\title{
Neurological Recovery in Conservatively Managed Severe Cervical Spondylotic Myelopathy: Case Report
}

\author{
Author \\ Hemali J. Gandhi \\ Advanced Physiotherapy and Sports Rehabilitation Department, Saifee Hospital \\ Email: drhemaligandhi@gmail.com, Mob: 9969985010
}

\begin{abstract}
Cervical spondylotic myelopathy is spinal cord compression due to pathophysiological narrowing of spinal canal causing ischemic and neuronal damage. Conservative approach in moderate to severe cervical spondylotic myelopathy is rare. We present a case study of a 51-year-old patient with complains of imbalance and weakness in bilateral lower limbs for 2 weeks along with difficulty in standing and walking. Magnetic Resonance Imaging revealed large posterior disc herniation compressing cervical spinal cord at level C5-C6 and ligamentum flavum hypertrophy with cord oedema. The patient underwent conservative treatment with subsequent resolution of symptoms. 2 years follow-up indicated no recurrence and complete resolution of clinical symptoms. This case report shows a possibility of conservative trial for moderate to severe cervical spondylotic myelopathy.

Keywords: Severe cervical spondylotic myelopathy, neurological recovery, conservative management.
\end{abstract}

\section{Introduction}

Cervical Spondylotic Myelopathy (CSM) is characterized by compression of the spinal cord due to pathophysiological narrowing of spinal canal secondary to degenerative or congenital changes ${ }^{(1)}$. Pathophysiological factors causing the narrowing of the spinal canal and leading to CSM are divided into 2 categories: a) Static: comprising of congenital stenosis and degenerative diseases. b) Dynamic: normal motion of cervical spine may aggravate spinal cord damage due to mechanical abnormalities of cervical spine. These pathophysiological processes lead to spinal cord compression causing chronic ischemia and neuronal damage ${ }^{(1)}$.

A cohort study of Eastern Asia reported prevalence of CSM caused hospitalization to be 4.04 per million person-years with higher prevalence in males and older patients compared to females and younger patients ${ }^{(2)}$. The best course of intervention for patients presenting with signs of CSM remains debatable. While severe and progressive cervical myelopathy is an indication for surgery, there is no conclusive agreement regarding treatment for mild and moderate non-progressive forms of CSM. Patients across all age-groups with severe and progressive CMS undergoing surgical decompression of cervical spine, anteriorly, posteriorly or circumferentially reported significant improvement $^{(3)}$.

However there is no consensus regarding treatment for mild and moderate non-progressive forms of CSM. A study by Matz et al concluded there is not 
enough data to determine whether surgery is the most indicated intervention for milder forms of $\mathrm{CSM}^{(1)}$. A prospective clinical trial comparing conservative versus surgical treatment in mild and moderate forms of CSM did not find significant difference in the outcomes for up to 10 years of follow up(1). Currently, to the best of our knowledge, there are no published reports describing treatment using physical therapy with emphasis on neural tissue mobilization and therapeutic exercise for patients with CSM. Therefore, the purpose of this study is to describe the outcomes of physical therapy treatment in a patient with severe CSM.

\section{Case Presentation}

A 51-year-old male patient presented with complaints of imbalance and weakness in bilateral lower limbs and difficulty in standing and walking since 2 weeks. Patient reported continuous sharp shooting cervical pain 3 weeks before the visit at C3-C7 level radiating to right upper limb till anterior aspect of medial 3 fingers which exacerbated on cervical movement. This cervical pain was followed by numbness, weakness, dryness and reduced sensations in the right hand. He also complained of mild difficulty in micturition with urine retention. Functionally, he had difficulty in writing, buttoning/unbuttoning his shirt and eating with a spoon.

Clinically patient presented with cervical list on the left side with right shoulder elevation. Superficial sensations were reduced at right C7-C8 dermatome (70\% reduction) with intact deep sensations and intact lower limb sensations. Myotomal weakness (muscle power grade- 2/5) was present at right C7, C8, T1 level. Clinical assessment revealed right triceps hyporeflexia, bilateral lower limb hyperreflexia, bilateral plantars upgoing, positive right Hoffman reflex, right ankle clonus and bilateral knee extensor hypertonia. Upper Limb Tension Test (ULTT) 1 was positive at 70 degrees of elbow flexion. Coordination tests were normal in upper limbs however affected in lower limbs with the right side being more affected than the left. Gait assessment revealed a wide stationed gait, right knee locking during stance phase and a positive Rhomberg test.

\section{Investigations}

Magnetic Resonance Imaging revealed large posterior disc herniation compressing cervical spinal cord at C5-C6 level with ligamentum flavum hypertrophy and cord oedema. Posterior disc herniation was also detected at C3-C4, C4-C5, C6C7 level. (Figure I)

\section{Treatment}

A comprehensive approach (1) was used in the initial treatment phase. The approach comprised of non thrust manual therapy (mechanical openers), Shacklock neural tissue mobilization (remote sliders using wrist and elbow components), symptomatic pain relief using 4pole interferential current therapy (neck and right upper limb), rigid cervical collar, intermittent bed rest and avoidance of high risk activities and risky environment along with medications (Trypsin96 Mg, Bromelain180 Mg, Rutoside $200 \mathrm{Mg}$, Pregabalin 300Mg).

One week after the comprehensive approach, cervical stabilisation exercises, postural correction, lower limb strengthening, gait training and progressive neural tissue mobilisation- local sliders using shoulder and elbow components were gradually incorporated(1) for 4 weeks. Aerobic exercise on static cycle for 15-20 minutes (velocity and level of difficulty increased as possible) was also included in the later part of the treatment phase. After 1 month the patient was on progressive neural tissue mobilisation- single ended tensioner using wrist component, balance training (1 leg standing, standing with eyes open and eyes closed, standing on unstable surface) and was able to walk on even surfaces for 30 minutes.

\section{Outcomes and Follow-Up}

Modified Japanese Orthopaedic Association Score (mJOA) $(1,4)$

mJOA is a clinical grading system proposed by Benzel et al which assesses motor dysfunction in the 
upper and lower extremities, sensory function in the upper extremities, and bladder function. This scale quantifies neurological function on a scale ranging from 0 (worst possible) to 18 (best possible) points (1). Fehlings et al. defined the severity of myelopathy as mild if the mJOA score is 15 and above, moderate if the mJOA score ranges from 12 to 14 and severe if the mJOA score is below $12^{[5]}$. Past studies report that the patient should score at least 12 points for the indication of nonsurgical treatment ${ }^{1}$.

\section{Nurick Scale}

The Nurick scale is a six-grade system and probably the most useful classification for stratifying the level of functional restriction in mobility based on difficulty in walking caused by cervical myelopathy. The scale ranges from 0 (no evidence of cord disease) to 5(bedridden /chair bound $)^{(6)}$.

\section{Neck Disability Index}

The Neck Disability Index (NDI) is a widely used 10-item questionnaire clinically proven to be valid and reliable for determining neck-related pain and disability. Scores can range from 0 (best possible) to 50 (worst possible) points (1).

\section{Berg Balance Scale}

The Berg Balance Scale (BBS) is a widely used gold standard functional test to measure a person's static and dynamic balance abilities. The test involves performing 14 simple balance related tasks, ranging from standing up from sitting position, to standing on one foot. The degree of success in achieving each task is given a score of zero (unable) to four (independent), and the final maximum score is the sum of scores for all items (maximum score bein 56). The scale ranges from 41-56- independent, 21-40- walking with assistance, 0-20- wheelchair bound. ${ }^{(7)}$

Patient was assessed at baseline, after 1week,1 month, 3 months, 1year and 2years from baseline for the above mentioned outcome measures.(table 1)Clinically on assessment within a week, the patient had improved sensation in C7-C8 dermatome $(20 \%$ reduction in superficial sensations), improved strength in C7,C8,T1 myotome (muscle power grade 3/5) and no bladder complaints. Examination revealed normal tone in left knee extensor, with persistent hypertonia in right knee ankle. ULTT 1 indicated symptoms at 40degrees of elbow flexion. At the end of 4weeks, reassessment revealed normal pinprick sensation in right upper limb, normal tone in bilateral lower limbs, ankle clonus and minimal weakness in right C7 myotome (muscle power grade- 4/5). Functionally, he was able to write, button his shirt and eat with a spoon. At 3months from baseline patient reported resolution of pain in the neck, right upper limb and bilateral lower limbs and functionally he was able to perform all the activities which he reported difficulty performing at baseline. Neurological assessment revealed improved coordination, improved gait, balance with occasional loss of balance on sudden turning. The patient was contacted after1year and 2years of his treatment to assess his functional status. He had good neurological recovery, with no reversal of clinical signs or symptoms after 2years. Patient precautionary avoided lifting heavy weights as reflected in NDI score (table1).

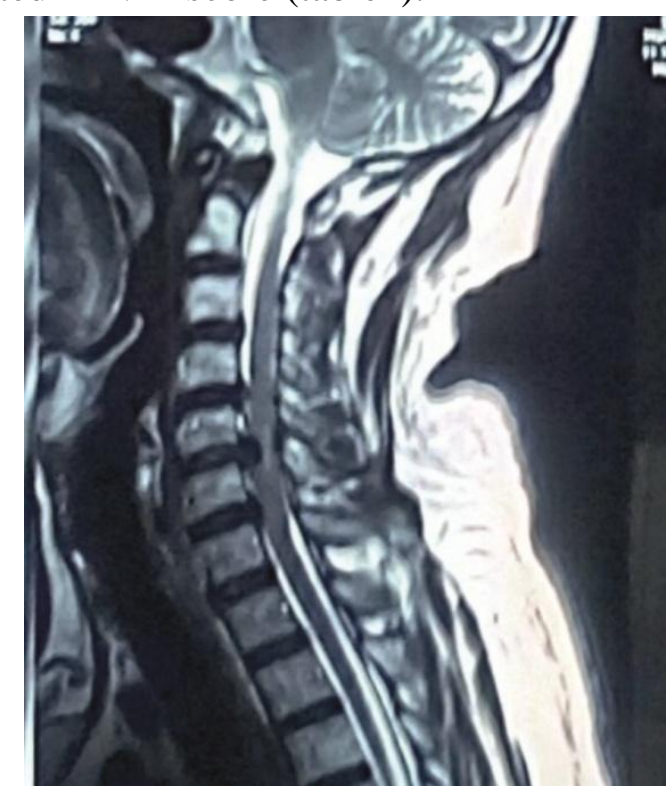

Figure I- Magnetic Resonance Imaging revealed large posterior disc herniation compressing cervical spinal cord at C5-C6 level with ligamentum flavum hypertrophy and cord edema. 
Table 1 Patients progressive neurological and functional status scores on Outcome Scales

\begin{tabular}{|l|c|c|c|c|}
\hline $\begin{array}{l}\text { Outcome } \\
\text { Measures }\end{array}$ & $\begin{array}{c}\text { Modified Japanese Orthopaedic } \\
\text { Association Score }\end{array}$ & Nurick Scale & Neck Disbility Index & Berg Balance Scale \\
\hline Baseline Score & $11 / 18$ & Grade 3 & $32 / 50=64 \%$ & $35 / 56$ \\
\hline Post 1week & $13 / 18$ & Grade 3 & $28 / 50=56 \%$ & $42 / 56$ \\
\hline Post 4weeks & $15 / 18$ & Grade 2 & $18 / 50=26 \%$ & $48 / 56$ \\
\hline Post 3months & $17 / 18$ & Grade 1 & $6 / 50=12 \%$ & $52 / 56$ \\
\hline Post 1year & $18 / 18$ & Grade 0 & $4 / 50=8 \%$ & $56 / 56$ \\
\hline Post 2years & $18 / 18$ & Grade 0 & $4 / 50=8 \%$ & $56 / 56$ \\
\hline
\end{tabular}

\section{Discussion}

The case report demonstrated that physical therapy treatment with emphasis on manual therapy(neural tissue mobilisation) and therapeutic exercise achieved satisfactory results after 2 years in a patient with severe CSM. The treatment strategy was to reduce pain and paresthesia, improve neuromuscular control of the deep neck muscles, strengthen the upper and lower limbs and improve both proprioception and aerobic fitness.

One mechanism for neck pain spreading down the arm and cervical myelopathy is cord oedema and the sensitisation of neural tissues ${ }^{(8)}$. Cord oedema with degenerated stenosis of the spine aggravates neural compression. Inflammation can ultimately lead to adhesions between the herniated disc and the nerve root thereby impairing gliding of the nerve root. In the acute and sub-acute stages of nerve root compression, neural conduction block, intraneural oedema, mechanical sensitisation and increase of sodium channel density have been reported $^{(9)}$. Dysfunction can also extend to primary sensory neurons within the dorsal root ganglion ${ }^{(9)}$. The result of these changes manifests itself as increased mechano-sensitivity.

Neural tissue management is a Physiotherapy intervention suggested for nerve-related neck and arm pain ${ }^{(8)}$. It uses specific positions and movements of the neck and arm to resolve symptoms, reduces nerve mechano-sensitivity, and restores function ${ }^{(8)}$. Mechanical openers involve positioning the neck in slight flexion and lateral flexion thereby increasing the space in the spinal canal and reducing cord compression.
These techniques that produce dynamic and static opening of the bony and fascial interface around the nerve root $^{(8,9)}$ have been proposed for reduction of nerve root mechano-sensitivity.

Neural tissue mobilisation can be performed in opening positions. They involve a specific sequence of joint movements during which the therapist lengthens the nerve at one joint and simultaneously reduces its length at an adjacent joint to produce sliding movements of neural structures relative to adjacent tissues. These are called sliders or gliding techniques. A slightly more aggressive maneuver is the tensioning technique which increases the distance between the two ends of the nerve tract in an oscillatory fashion ${ }^{(8,9)}$. These techniques can be used to potentially rehabilitate normal function of the nervous system.

Due to antero-posterior instability of the vertebral bodies of a degenerative nature, vertebral segment stabilisation of the cervical spine improves neuromuscular control of the deep neck muscles thereby improving the dynamic stability of cervical spine $^{(1)}$.

Almeida et al (2013) described the treatment of a 58 year-old patient with clinical signs of cervical myelopathy. The authors used a combined approach consisting of non-thrust manual therapy to the cervical spine, cervical stabilisation exercises, upper and lower extremity strengthening, balance exercises, and aerobic conditioning. While complete resolution of symptoms was not achieved, overall functional improvements and diminished pain levels were noted and maintained at 6-month follow-up ${ }^{(1)}$ 
The lack of more satisfactory results with surgery compared to conservative treatment ${ }^{(1)}$ increases the indication of physical therapy as a mode of treatment. However, we are yet to come across any studies using Shacklock neural tissue mobilisation and therapeutic exercise in severe CSM. Additional studies are needed to better understand the effects of neural mobilisation and therapeutic exercises for severe CSM.

\section{Conclusion}

This paper reported a case-study of a patient with severe cervical spondylotic myelopathy without rapidly progressing neurological impairments treated conservatively and documented satisfactory results using reliable and clinically valid outcome measures.

\section{Key Points}

-Conservative treatment focused on neural tissue mobilisation and therapeutic exercises are an effective treatment for symptomatic severe cervical spondylotic myelopathy.

-Outcome measures should be used for close monitoring of patient's symptoms during the treatment.

\section{References}

1. Gabriel Peixoto Lea o Almeida, Kysia Karine Almeida Carneiro, Amélia Pasqual Marques, Manual therapy and therapeutic exercise in patient with symptomatic cervical spondylotic myelopathy: A case report, Journal of Bodywork \& Movement Therapies (2013) 17, 504e509.

2. Wu JC1, Ko CC, et al, Epidemiology of cervical spondylotic myelopathy and its risk of causing spinal cord injury: a national cohort study. Neurosurg Focus. 2013 Jul;35(1):E10. Doi: 10.3171/2013.4.

3. Elizabeth Sateren Zoller, Dominic Cannella,et al, Diagnosis and medical and surgical management of cervical spondylotic myelopathy, Journal of the American Academy of Physician Assistants , 2015.

4. Kato S, Oshima Y, Oka H, et al. (2015) Comparison of the Japanese Orthopaedic Association (JOA) Score and Modified JOA (mJOA) Score for the Assessment of Cervical Myelopathy: A Multicenter Observational Study. PLoS ONE 10(4): e0123022. doi:10.1371/ journal. pone. 0123022.

5. Fehlings MG, Wilson JR, et al. Efficacy and safety of surgical decompression in patients with cervical spondylotic myelopathy: results of the AO Spine North America prospective multi-center study. J Bone Joint Surg Am. 2013; 95:1651-8. doi:

10.2106/JBJS.L. 00589PMID:24048552.

6. Kumbhar Kartik Revanappa, Vedantam Rajshekhar, Comparison of Nurick grading system and modified Japanese Orthopaedic Association scoring system in evaluation of patients with cervical spondylotic myelopathy, Eur Spine J (2011) 20:1545-1551 DOI 10.1007s00586-011-1773-y.

7. Berg K, Wood-Douphinee S. et al, Measuring balance in the elderly, validation of an instrument. Can. J. Pub. Health(1992), July/Aug S7-11.

8. Nee et al, Neural tissue management provides immediate clinically relevant benefits without harmful effects for patients with nerve related neck and arm pain: a randomised trial. Journal of Physiotherapy, 2012.

9. Efstathia, M.A et al, Effectiveness of neural mobilisation in patients with spinal radiculopathy: A critical review. Journal of Bodywork and Movement Therapy, 2014. 